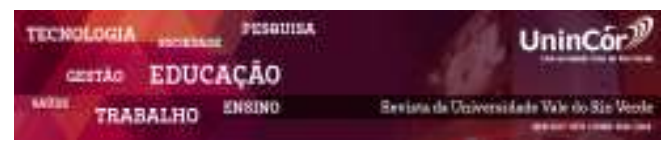

Revista da Universidade Vale do Rio Verde ISSN: 1517-0276 / EISSN: 2236-5362 Vol. 16 | n. 2 | Ano 2018

Jocias Maier Zanatta

Universidade Federal de Santa Maria - UFSM josk85@hotmail.com

\section{ANÁLISE DE PROPOSTA DE LOCAÇÃO DE IMPRESSORA DE EXAMES PARA SERVIÇO DE DIAGNÓSTICO POR IMAGEM EM HOSPITAL DE PEQUENO PORTE: PERSPECTIVA FINANCEIRA}

\section{RESUMO}

Diante da singularidade e complexidade de uma instituição hospitalar, sendo o serviço de diagnóstico por imagem primordial, faz-se necessário constantes estudos sob a perspectiva financeira. Este estudo tem como objetivo realizar a análise do impacto financeiro da mudança do processo de impressão de exames em serviço de diagnóstico por imagem em hospital de pequeno porte do Estado do Rio Grande do Sul. Busca-se elucidar a seguinte pergunta de pesquisa: Qual o impacto financeiro da aceitação de proposta de locação de impressora de exames em serviço de diagnóstico por imagem em hospital de pequeno porte? Para tanto, a pesquisa enquadrou-se em relação a abordagem como quantitativa, quanto aos procedimentos, uma pesquisa bibliográfica, descritiva e estudo de caso, e como técnicas de coleta e análise de dados utilizou-se pesquisa documental e planilha eletrônica. Conclui-se com o estudo, que a mudança no processo de impressão de exames substituindo o filme radiológico pelo papel fotográfico é viável, onde no período pesquisado representaria um ganho superior a $\mathrm{R} \$ 30.000,00$ (trinta mil reais) a instituição.

Palavras-chave: Hospital de pequeno porte; Serviço de diagnóstico por imagem; Impressora de exames; Perspectiva financeira.

\section{ANALYSIS OF PRINTER LEASE PROPOSAL FOR IMAGING DIAGNOSTIC SERVICE IN SMALL HOSPITAL: FINANCIAL PERSPECTIVE}

\begin{abstract}
Given the uniqueness and complexity of a hospital institution, being the primary imaging diagnostic service, it is necessary to carry out constant studies from a financial perspective. The objective of this study is to analyze the financial impact of the change in the process of printing the exams in diagnostic imaging services in a small hospital in the State of Rio Grande do Sul. The following research question is elucidated: What is the financial impact the acceptance of a proposal to lease an exam printer at an imaging diagnostic service in a small hospital? For this, the research was framed in relation to the quantitative approach, regarding the procedures, a bibliographical, descriptive and case study research, and as data collection and analysis techniques we used documentary research and spreadsheet. It is concluded with the study that the change in the printing process of exams replacing the radiological film with photographic paper is viable, where in the period studied it would represent a gain of more than
\end{abstract}


Keywords: Small hospital; Diagnostic imaging service; Exam printer; Financial perspective.

\section{INTRODUÇÃO}

Nas organizações, o processo de tomada de decisão se dá pela escolha de uma opção ou mais dentre as alternativas existentes, seguindo um roteiro de análise previamente definido para alcançar os objetivos propostos (ABDULLAH, 2013; HERRERA-VIEDMA; GARCÍALAPRESTA, 2014).

A área da saúde, em especial os hospitais, caracterizam-se por instituições de gestão complexa, frente as dificuldades financeiras e natureza dos serviços prestados (SILVA, 2014). Neste sentido, deve-se estar atendo as mudanças do mercado e considerar o contexto de fatores que modificam as práticas clínicas, demanda, custos, entre outros (BORBA; NETO, 2008).

Este trabalho busca realizar a análise do impacto financeiro da mudança do processo de impressão de exames em serviço de diagnóstico por imagem em hospital de pequeno porte do Estado do Rio Grande do Sul.

O hospital em questão é uma instituição hospitalar filantrópica, com características de hospital geral, de pequeno porte, com 51 leitos, 63 funcionários, e 19 médicos, localizada no Estado do Rio Grande do Sul. No serviço de diagnóstico por imagem, foram considerados os exames que possuem impressão em filme radiológico, que são eles: mamografia, tomografia, raio x e ultrassonografia.

Como problemática de pesquisa tem-se:

Qual o impacto financeiro da aceitação de proposta de locação de impressora de exames em serviço de diagnóstico por imagem em hospital de pequeno porte?

O presente estudo está estruturado em cinco partes, sendo que após a introdução, apresenta-se o caminho metodológico, seguido do referencial teórico sobre os assuntos abordados. Após, é apresentado por meio de tabelas o detalhamento da análise financeira realizada, e por fim, as considerações finais do estudo.

\section{METODOLOGIA}

Para Jung (2004), a metodologia é um conjunto de técnicas e procedimentos que tem como finalidade viabilizar a execução da pesquisa. Quanto ao caminho metodológico, o presente estudo em relação a abordagem tem característica quantitativa; como procedimentos, pesquisa bibliográfica, descritiva e estudo de caso; e para coleta e análise de dados, pesquisa documental e planilha eletrônica.

O Quadro 1 sintetiza o roteiro da pesquisa: 
Quadro 1 - Roteiro de pesquisa

\begin{tabular}{|c|c|}
\hline Etapas 1 & $\begin{array}{ll}\text { Avaliação } & \text { da } \\
\text { necessidade } & \text { da } \\
\text { instituição e escolha } & \text { do } \\
\text { tema } & \end{array}$ \\
\hline Etapas 2 & $\begin{array}{l}\text { Levantamento da receita } \\
\text { e quantidades de exames } \\
\text { realizados }\end{array}$ \\
\hline Etapas 3 & $\begin{array}{l}\text { Levantamento dos custos } \\
\text { fixos }\end{array}$ \\
\hline Etapas 4 & $\begin{array}{l}\text { Levantamento do custo } \\
\text { variável (pagamento } \\
\text { médicos) }\end{array}$ \\
\hline Etapa 5 & $\begin{array}{l}\text { Levantamento dos custos } \\
\text { variáveis do processo } \\
\text { atual (insumos) }\end{array}$ \\
\hline Etapa 6 & $\begin{array}{lcr}\text { Apuração dos } & \text { custos } \\
\text { variáveis do novo } & \text { nocesso (insumos) } \\
\text { procen }\end{array}$ \\
\hline Etapa 7 & $\begin{array}{l}\text { Apuração do resultado } \\
\text { financeiro do processo } \\
\text { atual e do novo processo }\end{array}$ \\
\hline
\end{tabular}

Fonte: Elaborado pelo autor, 2018

A coleta de dados foi realizada na documentação contábil da instituição e foram considerados os doze meses do ano de 2017 (dois mil e dezessete), contemplando todos os convênios atendidos. O levantamento da produção de exames levou em consideração somente os serviços que realizam impressão em filme radiológico, que são eles: mamografia, tomografia, raio x e ultrassonografia. Já para o levantamento dos valores relativos ao novo processo (proposta de locação de impressora) utilizou-se como parâmetro o custo médio de três empresas fornecedoras do serviço, as quais os nomes serão mantidos em sigilo.

Foi utilizada planilha eletrônica para análise dos dados quantitativos, onde foi realizada a triangulação entre os seguintes dados: receita bruta, quantidade de exames, custos fixos, custos variáveis, resultados e viabilidade da proposta.

\section{SERVIÇO DE DIAGNÓSTICO POR IMAGEM}

Para Bisagni (2000), um centro de diagnóstico por imagem é composto pelos serviços de radiologia convencional, tomografia, ultrassonografia, hemodinâmica e ressonância magnética. $\mathrm{O}$ mesmo autor, ressalta que o serviço de diagnóstico por imagem é fundamental para o funcionamento de uma instituição hospitalar, pois permite eficiência nos diagnósticos clínicos e cirúrgicos dos pacientes.

\section{ADMINISTRAÇÃO FINANCEIRA}

A administração financeira trata das tarefas dos administradores que gerenciam organizações de qualquer natureza, sejam financeiras ou não, públicas ou privadas, grandes ou pequenas, e com ou sem fins lucrativos (GITMAN, 2004).

A administração financeira é uma ciência que tem o objetivo de gerenciar o processo de captação e aplicação de recursos de forma eficiente. $\mathrm{O}$ ambiente competitivo e dinâmico em que as organizações estão inseridas faz com que as empresas utilizem novas técnicas de administração financeira como forma de adaptação organizacional (CABRELLI, FERREIRA, 2007).

Neste contexto, a administração financeira ganha importância frente aos desafios da escassez de recursos, realidade operacional e de gestão das organizações (ASSAF NETO; LIMA, 2014). A administração financeira assume papel fundamental na análise da perspectiva financeira das organizações, sobretudo as hospitalares. 


\section{PROCESSO DECISÓRIO}

O processo decisório é tema central nos estudos organizacionais e na vivência dos gestores, principalmente em relação formulação e implementação de estratégias (RAMOS; TAKAHASHI; ROGRLIO, 2015).

Nas organizações, o processo de tomada de decisão ocorre pela análise e escolha das alternativas existentes para alcançar os objetivos propostos (ABDULLAH, 2013; HERRERAVIEDMA; GARCÍA-LAPRESTA, 2014).

Neste sentido, os gestores conduzem as organizações através das inúmeras tomadas de decisões e seus respectivos desdobramentos e resultados, onde quanto maior o número de alternativas possíveis mais complexo é o processo decisório (DACORSO, 2000; ZAMARIOLI, 2003).

\section{ANÁLISE E DISCUSSÃO DOS RESULTADOS}

Neste tópico será apresentado o diagnóstico do atual processo de impressão de exames, análise da proposta de locação de impressora de exames, e evidências da análise financeira.

\section{DIAGNÓSTICO DO ATUAL PROCESSO DE IMPRESSÃO DE EXAMES}

Inicialmente é apresentado o levantamento de dados referente a produção do serviço de diagnóstico por imagem (quantidade de exames e receita financeira) no período de janeiro a dezembro do ano de 2017 (dois mil e dezessete) referente a todos os atendimentos realizados independente do convênio.

A Tabela 1 apresenta o quantitativo de exames realizados.

Tabela 1 - Quantidade de exames

\begin{tabular}{lccccc}
\hline Mês & Mamografia & Tomografia & Ultrassonografia & Raio X & Total \\
\hline Janeiro & 81 & 49 & 228 & 278 & 636 \\
Fevereiro & 91 & 48 & 204 & 268 & 611 \\
Março & 107 & 69 & 268 & 417 & 861 \\
Abril & 80 & 81 & 324 & 318 & 803 \\
Maio & 91 & 72 & 353 & 427 & 943 \\
Junho & 78 & 48 & 231 & 407 & 764 \\
Julho & 87 & 72 & 331 & 392 & 882 \\
Agosto & 82 & 105 & 409 & 540 & 1.136 \\
Setembro & 91 & 73 & 282 & 411 & 857 \\
Outubro & 94 & 89 & 297 & 363 & 843 \\
Novembro & 108 & 76 & 367 & 391 & 942 \\
Dezembro & 98 & 50 & 303 & 282 & 733 \\
\hline Total & $\mathbf{1 . 0 8 8}$ & $\mathbf{8 3 2}$ & $\mathbf{3 . 5 9 7}$ & $\mathbf{4 . 4 9 4}$ & $\mathbf{1 0 . 0 1 1}$ \\
\hline
\end{tabular}

Fonte: Elaborado pelo autor, 2018.

A Tabela 2 apresenta a receita bruta referente ao período pesquisado, contendo todos os exames realizados, independente do convênio do paciente. 
Tabela 2 - Receita bruta

\begin{tabular}{|c|c|c|c|c|c|}
\hline Mês & Mamografia & Tomografia & Ultrassonografia & Raio X & Total \\
\hline Janeiro & $\mathrm{R} \$ 4.483,54$ & $\mathrm{R} \$ 11.465,75$ & $\mathrm{R} \$ 18.456,52$ & $\mathrm{R} \$ 5.173,89$ & $\mathrm{R} \$ 39.579,70$ \\
\hline Fevereiro & $\mathrm{R} \$ 4.263,21$ & $\mathrm{R} \$ 10.343,47$ & $\mathrm{R} \$ 14.649,80$ & $\mathrm{R} \$ 4.981,02$ & $\mathrm{R} \$ 34.237,50$ \\
\hline Março & $\mathrm{R} \$ 5.901,68$ & $\mathrm{R} \$ 8.010,43$ & $\mathrm{R} \$ 20.804,35$ & $\mathrm{R} \$ 9.006,85$ & $\mathrm{R} \$ 53.723,31$ \\
\hline Abril & $\mathrm{R} \$ 4.409,61$ & $\mathrm{R} \$ 19.569,07$ & $\mathrm{R} \$ 24.680,84$ & $\mathrm{R} \$ 6.570,46$ & $\mathrm{R} \$ 55.229,98$ \\
\hline Maio & $\mathrm{R} \$ 4.798,49$ & $\mathrm{R} \$ 18.505,34$ & $\mathrm{R} \$ 27.079,01$ & $\mathrm{R} \$ 9.900,25$ & $\mathrm{R} \$ 60.283,09$ \\
\hline Junho & $\mathrm{R} \$ 4.481,61$ & $\mathrm{R} \$ 11.266,85$ & $\mathrm{R} \$ 19.567,80$ & $\mathrm{R} \$ 8.963,13$ & $\mathrm{R} \$ 44.279,39$ \\
\hline Julho & $\mathrm{R} \$ 4.617,90$ & $\mathrm{R} \$ 18.386,81$ & $\mathrm{R} \$ 26.058,75$ & $\mathrm{R} \$ 8.031,79$ & $\mathrm{R} \$ 57.095,25$ \\
\hline Agosto & $\mathrm{R} \$ 4.767,89$ & $\mathrm{R} \$ 27.815,26$ & $\mathrm{R} \$ 33.423,18$ & $\mathrm{R} \$ 10.441,62$ & $\mathrm{R} \$ 76.447,95$ \\
\hline Setembro & $\mathrm{R} \$ 4.778,11$ & $\mathrm{R} \$ 15.319,30$ & $\mathrm{R} \$ 23.400,01$ & $\mathrm{R} \$ 8.565,17$ & $\mathrm{R} \$ 52.062,59$ \\
\hline Outubro & $\mathrm{R} \$ 5.518,85$ & $\mathrm{R} \$ 21.367,11$ & $\mathrm{R} \$ 25.524,11$ & $\mathrm{R} \$ 6.897,46$ & $\mathrm{R} \$ 59.307,53$ \\
\hline Novembro & $\mathrm{R} \$ 6.161,78$ & $\mathrm{R} \$ 19.316,03$ & $\mathrm{R} \$ 30.896,59$ & $\mathrm{R} \$ 7.815,52$ & $\mathrm{R} \$ 64.189,92$ \\
\hline Dezembro & $\mathrm{R} \$ 4.953,47$ & $\mathrm{R} \$ 11.225,91$ & $\mathrm{R} \$ 23.173,46$ & $\mathrm{R} \$ 4.510,97$ & $\mathrm{R} \$ 43.863,81$ \\
\hline Total & R\$ 59.136,14 & $\mathrm{R} \$ 202.591,33$ & $\mathrm{R} \$ 287.714,42$ & $\mathrm{R} \$ 90.858,13$ & $\mathrm{R} \$ 640.300,02$ \\
\hline
\end{tabular}

No levantamento dos custos fixos foram considerados três técnicos de radiologia, com piso salarial da categoria (dos salários mínimos nacionais), insalubridade de $40 \%$ (quarenta por cento), vale alimentação e contribuições sociais.
Também, foi realizado rateio proporcional a utilização do serviço referente a energia elétrica, água, internet e manutenção preventiva dos equipamentos.

A Tabela 3 apresenta os custos fixos.

Tabela 3 - Custos fixos

\begin{tabular}{|c|c|c|c|c|c|}
\hline Mês & Funcionários & AgualLuz & $\begin{array}{c}\text { Manutenção Preventi- } \\
\text { va } \\
\end{array}$ & Internet & Total \\
\hline Janeiro & $\mathrm{R} \$ 4.098,82$ & $\mathrm{R} \$ 4.644,39$ & $\mathrm{R} \$ 420,00$ & $\mathrm{R} \$ 133,33$ & $\mathrm{R} \$ 9.296,55$ \\
\hline Fevereiro & $\mathrm{R} \$ 4.098,82$ & $\mathrm{R} \$ 4.644,39$ & $\mathrm{R} \$ 420,00$ & $\mathrm{R} \$ 133,33$ & $\mathrm{R} \$ 9.296,55$ \\
\hline Março & $\mathrm{R} \$ 4.098,82$ & $\mathrm{R} \$ 4.644,39$ & $\mathrm{R} \$ 420,00$ & $\mathrm{R} \$ 133,33$ & $\mathrm{R} \$ 9.296,55$ \\
\hline Abril & $\mathrm{R} \$ 4.098,82$ & $\mathrm{R} \$ 4.644,39$ & $\mathrm{R} \$ 420,00$ & $\mathrm{R} \$ 133,33$ & $\mathrm{R} \$ 9.296,55$ \\
\hline Maio & $\mathrm{R} \$ 4.098,82$ & $\mathrm{R} \$ 4.644,39$ & $\mathrm{R} \$ 420,00$ & $\mathrm{R} \$ 133,33$ & $\mathrm{R} \$ 9.296,55$ \\
\hline Junho & $\mathrm{R} \$ 4.098,82$ & $\mathrm{R} \$ 4.644,39$ & $\mathrm{R} \$ 420,00$ & $\mathrm{R} \$ 133,33$ & $\mathrm{R} \$ 9.296,55$ \\
\hline Julho & $\mathrm{R} \$ 4.098,82$ & $\mathrm{R} \$ 4.644,39$ & $\mathrm{R} \$ 420,00$ & $\mathrm{R} \$ 133,33$ & $\mathrm{R} \$ 9.296,55$ \\
\hline Agosto & $\mathrm{R} \$ 4.098,82$ & $\mathrm{R} \$ 4.644,39$ & $\mathrm{R} \$ 420,00$ & $\mathrm{R} \$ 133,33$ & $\mathrm{R} \$ 9.296,55$ \\
\hline Setembro & $\mathrm{R} \$ 4.098,82$ & $\mathrm{R} \$ 4.644,39$ & $\mathrm{R} \$ 420,00$ & $\mathrm{R} \$ 133,33$ & $\mathrm{R} \$ 9.296,55$ \\
\hline Outubro & $\mathrm{R} \$ 4.098,82$ & $\mathrm{R} \$ 4.644,39$ & $\mathrm{R} \$ 420,00$ & $\mathrm{R} \$ 133,33$ & $\mathrm{R} \$ 9.296,55$ \\
\hline Novembro & $\mathrm{R} \$ 4.098,82$ & $\mathrm{R} \$ 4.644,39$ & $\mathrm{R} \$ 420,00$ & $\mathrm{R} \$ 133,33$ & $\mathrm{R} \$ 9.296,55$ \\
\hline Dezembro & $\mathrm{R} \$ 4.098,82$ & $\mathrm{R} \$ 4.644,39$ & $\mathrm{R} \$ 420,00$ & $\mathrm{R} \$ 133,33$ & $\mathrm{R} \$ 9.296,55$ \\
\hline Total & $\mathrm{R} \$ \mathbf{4 9 . 1 8 5 , 8 8}$ & $\mathrm{R} \$ 55.732,69$ & $\mathrm{R} \$ \mathbf{5 . 0 4 0 , 0 0}$ & $\mathrm{R} \$ 1.600,00$ & $\mathrm{R} \$ \mathbf{1 1 1 . 5 5 8 , 5 8}$ \\
\hline
\end{tabular}

Fonte: Elaborado pelo autor, 2018.

No serviço de diagnóstico por imagem da instituição, o pagamento aos profissionais médicos referente aos exames realizados e laudados são feitos na seguinte proporção em relação a receita bruta: mamografia (30\%), tomografia $(35 \%)$, ultrassonografia $(50 \%)$ e raio $\mathrm{x}(25 \%)$.

A Tabela 4 demonstra o custo variável com os profissionais médicos referente ao período pesquisado. 
Tabela 4 - Custos variáveis - profissionais médicos

\begin{tabular}{|c|c|c|c|c|c|}
\hline Mês & Mamografia & Tomografia & Ultrassonografia & Raio X & Total \\
\hline Janeiro & $\mathrm{R} \$ 1.345,06$ & $\mathrm{R} \$ 4.013,01$ & $\mathrm{R} \$ 9.228,26$ & $\mathrm{R} \$ 1.293,47$ & $\mathrm{R} \$ 15.879,81$ \\
\hline Fevereiro & $\mathrm{R} \$ 1.278,96$ & $\mathrm{R} \$ 3.620,21$ & $\mathrm{R} \$ 7.324,90$ & $\mathrm{R} \$ 1.245,26$ & $\mathrm{R} \$ 13.469,33$ \\
\hline Março & $\mathrm{R} \$ 1.770,50$ & $\mathrm{R} \$ 6.303,65$ & $\mathrm{R} \$ 10.402,18$ & $\mathrm{R} \$ 2.251,71$ & $\mathrm{R} \$ 20.728,04$ \\
\hline Abril & $\mathrm{R} \$ 1.322,88$ & $\mathrm{R} \$ 6.849,17$ & $\mathrm{R} \$ 12.340,42$ & $\mathrm{R} \$ 1.642,62$ & $\mathrm{R} \$ 22.155,09$ \\
\hline Maio & $\mathrm{R} \$ 1.439,55$ & $\mathrm{R} \$ 6.476,87$ & $\mathrm{R} \$ 13.539,51$ & $\mathrm{R} \$ 2.475,06$ & $\mathrm{R} \$ 23.930,98$ \\
\hline Junho & $\mathrm{R} \$ 1.344,48$ & $\mathrm{R} \$ 3.943,40$ & $\mathrm{R} \$ 9.783,90$ & $\mathrm{R} \$ 2.240,78$ & $\mathrm{R} \$ 17.312,56$ \\
\hline Julho & $\mathrm{R} \$ 1.385,37$ & $\mathrm{R} \$ 6.435,38$ & $\mathrm{R} \$ 13.029,38$ & $\mathrm{R} \$ 2.007,95$ & $\mathrm{R} \$ 22.858,08$ \\
\hline Agosto & $\mathrm{R} \$ 1.430,37$ & $\mathrm{R} \$ 9.735,34$ & $\mathrm{R} \$ 16.711,59$ & $\mathrm{R} \$ 2.610,41$ & $\mathrm{R} \$ 30.487,70$ \\
\hline Setembro & $\mathrm{R} \$ 1.433,43$ & $\mathrm{R} \$ 5.361,76$ & $\mathrm{R} \$ 11.700,01$ & $\mathrm{R} \$ 2.141,29$ & $\mathrm{R} \$ 20.636,49$ \\
\hline Outubro & $\mathrm{R} \$ 1.655,66$ & $\mathrm{R} \$ 7.478,49$ & $\mathrm{R} \$ 12.762,06$ & $\mathrm{R} \$ 1.724,37$ & $\mathrm{R} \$ 23.620,56$ \\
\hline Novembro & $\mathrm{R} \$ 1.848,53$ & $\mathrm{R} \$ 6.760,61$ & $\mathrm{R} \$ 15.448,30$ & $\mathrm{R} \$ 1.953,88$ & $\mathrm{R} \$ 26.011,32$ \\
\hline Dezembro & $\mathrm{R} \$ 1.486,04$ & $\mathrm{R} \$ 3.929,07$ & $\mathrm{R} \$ 11.586,73$ & $\mathrm{R} \$ 1.127,74$ & $\mathrm{R} \$ 18.129,58$ \\
\hline Total & R\$ 17.740,84 & R\$ 70.906,97 & R\$ $143.857,21$ & $R \$ 22.714,53$ & R\$ 255.219,55 \\
\hline
\end{tabular}

Da mesma forma, foram apurados os custos variáveis realizados no período pesquisado, onde considerou-se os insumos para impressão (filmes radiológicos), insumos para aplicação de contrastes (medicamentos), manutenções corretivas realizadas e materiais diversos (expediente, limpeza e informática).
Importante ressaltar, que de acordo com o Código Tributário Municipal, a instituição hospitalar não tem incidência do Imposto Sobre Serviços de Qualquer Natureza (ISSQN).

A Tabela 5 demonstra os custos variáveis com insumos.

Tabela 5 - Custos variáveis - insumos

\begin{tabular}{|c|c|c|c|c|c|}
\hline Mês & Insumos - Impressão & Insumos - Contrastes & $\begin{array}{c}\text { Manutenção correti- } \\
\text { va }\end{array}$ & Outros materiais & Total \\
\hline Janeiro & $\mathrm{R} \$ 5.543,58$ & $\mathrm{R} \$ 2.160,75$ & $\mathrm{R} \$ 520,40$ & $\mathrm{R} \$ 558,33$ & $\mathrm{R} \$ 8.783,06$ \\
\hline Fevereiro & $\mathrm{R} \$ 5.543,58$ & $\mathrm{R} \$ 2.160,75$ & $\mathrm{R} \$ 520,40$ & $\mathrm{R} \$ 558,33$ & $\mathrm{R} \$ 8.783,06$ \\
\hline Março & $\mathrm{R} \$ 5.543,58$ & $\mathrm{R} \$ 2.160,75$ & $\mathrm{R} \$ 520,40$ & $\mathrm{R} \$ 558,33$ & $\mathrm{R} \$ 8.783,06$ \\
\hline Abril & $\mathrm{R} \$ 5.543,58$ & $\mathrm{R} \$ 2.160,75$ & $\mathrm{R} \$ 520,40$ & $\mathrm{R} \$ 558,33$ & $\mathrm{R} \$ 8.783,06$ \\
\hline Maio & $\mathrm{R} \$ 5.543,58$ & $\mathrm{R} \$ 2.160,75$ & $\mathrm{R} \$ 520,40$ & $\mathrm{R} \$ 558,33$ & $\mathrm{R} \$ 8.783,06$ \\
\hline Junho & $\mathrm{R} \$ 5.543,58$ & $\mathrm{R} \$ 2.160,75$ & $\mathrm{R} \$ 520,40$ & $\mathrm{R} \$ 558,33$ & $\mathrm{R} \$ 8.783,06$ \\
\hline Julho & $\mathrm{R} \$ 5.543,58$ & $\mathrm{R} \$ 2.160,75$ & $\mathrm{R} \$ 520,40$ & $\mathrm{R} \$ 558,33$ & $\mathrm{R} \$ 8.783,06$ \\
\hline Agosto & $\mathrm{R} \$ 5.543,58$ & $\mathrm{R} \$ 2.160,75$ & $\mathrm{R} \$ 520,40$ & $\mathrm{R} \$ 558,33$ & $\mathrm{R} \$ 8.783,06$ \\
\hline Setembro & $\mathrm{R} \$ 5.543,58$ & $\mathrm{R} \$ 2.160,75$ & $\mathrm{R} \$ 520,40$ & $\mathrm{R} \$ 558,33$ & $\mathrm{R} \$ 8.783,06$ \\
\hline Outubro & $\mathrm{R} \$ 5.543,58$ & $\mathrm{R} \$ 2.160,75$ & $\mathrm{R} \$ 520,40$ & $\mathrm{R} \$ 558,33$ & $\mathrm{R} \$ 8.783,06$ \\
\hline Novembro & $\mathrm{R} \$ 5.543,58$ & $\mathrm{R} \$ 2.160,75$ & $\mathrm{R} \$ 520,40$ & $\mathrm{R} \$ 558,33$ & $\mathrm{R} \$ 8.783,06$ \\
\hline Dezembro & $\mathrm{R} \$ 5.543,58$ & $\mathrm{R} \$ 2.160,75$ & $\mathrm{R} \$ 520,40$ & $\mathrm{R} \$ 558,33$ & $\mathrm{R} \$ 8.783,06$ \\
\hline Total & $\mathrm{R} \$ \mathbf{6 6 . 5 2 2 , 9 7}$ & R\$ 25.929,00 & $\mathrm{R} \$ \mathbf{6 . 2 4 4 , 8 2}$ & R\$ 6.699,98 & R\$ 105.396,78 \\
\hline
\end{tabular}


A partir dos dados coletados elaborou-se o demonstrativo de resultado financeiro do atual processo de impressão de exames que é realizado com a utilização de filme radiológico.

A Tabela 6 ilustra o demonstrativo de resultado financeiro.

Tabela 6 - Resultado financeiro - atual processo (filme radiológico)

\begin{tabular}{|c|c|}
\hline Descrição & Valor \\
\hline Receita & $\mathrm{R} \$ 640.300,02$ \\
\hline Custo Fixo & $\mathrm{R} \$ 111.558,58$ \\
\hline Custo Variável (médico) & $\mathrm{R} \$ 255.219,55$ \\
\hline Custo Variável (insumos) & $\mathrm{R} \$ 105.396,78$ \\
\hline Resultado Anual & $\begin{array}{c}\text { R\$ } \\
168.125,11 \\
\end{array}$ \\
\hline Resultado Mensal & $R \$ 14.010,43$ \\
\hline
\end{tabular}

\section{ANÁliSE DE PROPOSTA DE LOCAÇÃO DE IMPRESSORA DE EXAMES}

A intenção em analisar a perspectiva financeira de uma proposta para novo processo de impressão de exames que substitui o filme radiológico pelo papel fotográfico, é entregar informações aos gestores para a tomada de decisão.

Para esta análise foram mantidos os dados inicias apresentados referentes a Tabela 1 (quantidade de exames), Tabela 2 (receita bruta), Tabela 3 (custos fixos) e Tabela 4 (custos variáveis - profissionais médicos), pois entendese que o novo processo tem impacto apenas nos custos variáveis de insumos e no resultado financeiro.

Para o cálculo dos custos variáveis realizados no período pesquisado, onde considerou-se os insumos para impressão (impressão em papel fotográfico), insumos para aplicação de contrastes (medicamentos), manutenções corretivas realizadas e materiais diversos (expediente, limpeza e informática).

A Tabela 7 apresenta os custos variáveis.

Tabela 7 - Custos variáveis - insumos

\begin{tabular}{|c|c|c|c|c|c|}
\hline Mês & Insumos - Impressão & Insumos - Contrastes & $\begin{array}{c}\text { Manutenção correti- } \\
\text { va }\end{array}$ & $\begin{array}{c}\text { Outros Materi- } \\
\text { ais }\end{array}$ & Total \\
\hline Janeiro & $\mathrm{R} \$ 2.970,00$ & $\mathrm{R} \$ 2.160,75$ & $\mathrm{R} \$ 520,40$ & $\mathrm{R} \$ 558,33$ & $\mathrm{R} \$ 6.209,48$ \\
\hline Fevereiro & $\mathrm{R} \$ 2.970,00$ & $\mathrm{R} \$ 2.160,75$ & $\mathrm{R} \$ 520,40$ & $\mathrm{R} \$ 558,33$ & $\mathrm{R} \$ 6.209,48$ \\
\hline Março & $\mathrm{R} \$ 2.970,00$ & $\mathrm{R} \$ 2.160,75$ & $\mathrm{R} \$ 520,40$ & $\mathrm{R} \$ 558,33$ & $\mathrm{R} \$ 6.209,48$ \\
\hline Abril & $\mathrm{R} \$ 2.970,00$ & $\mathrm{R} \$ 2.160,75$ & $\mathrm{R} \$ 520,40$ & $\mathrm{R} \$ 558,33$ & $\mathrm{R} \$ 6.209,48$ \\
\hline Maio & $\mathrm{R} \$ 2.970,00$ & $\mathrm{R} \$ 2.160,75$ & $\mathrm{R} \$ 520,40$ & $\mathrm{R} \$ 558,33$ & $\mathrm{R} \$ 6.209,48$ \\
\hline Junho & $\mathrm{R} \$ 2.970,00$ & $\mathrm{R} \$ 2.160,75$ & $\mathrm{R} \$ 520,40$ & $\mathrm{R} \$ 558,33$ & $\mathrm{R} \$ 6.209,48$ \\
\hline Julho & $\mathrm{R} \$ 2.970,00$ & $\mathrm{R} \$ 2.160,75$ & $\mathrm{R} \$ 520,40$ & $\mathrm{R} \$ 558,33$ & $\mathrm{R} \$ 6.209,48$ \\
\hline Agosto & $\mathrm{R} \$ 2.970,00$ & $\mathrm{R} \$ 2.160,75$ & $\mathrm{R} \$ 520,40$ & $\mathrm{R} \$ 558,33$ & $\mathrm{R} \$ 6.209,48$ \\
\hline Setembro & $\mathrm{R} \$ 2.970,00$ & $\mathrm{R} \$ 2.160,75$ & $\mathrm{R} \$ 520,40$ & $\mathrm{R} \$ 558,33$ & $\mathrm{R} \$ 6.209,48$ \\
\hline Outubro & $\mathrm{R} \$ 2.970,00$ & $\mathrm{R} \$ 2.160,75$ & $\mathrm{R} \$ 520,40$ & $\mathrm{R} \$ 558,33$ & $\mathrm{R} \$ 6.209,48$ \\
\hline Novembro & $\mathrm{R} \$ 2.970,00$ & $\mathrm{R} \$ 2.160,75$ & $\mathrm{R} \$ 520,40$ & $\mathrm{R} \$ 558,33$ & $\mathrm{R} \$ 6.209,48$ \\
\hline Dezembro & $\mathrm{R} \$ 2.970,00$ & $\mathrm{R} \$ 2.160,75$ & $\mathrm{R} \$ 520,40$ & $\mathrm{R} \$ 558,33$ & $\mathrm{R} \$ 6.209,48$ \\
\hline Total & R\$ 35.640,00 & R\$ 25.929,00 & $\mathrm{R} \$ 6.244,82$ & $\mathrm{R} \$ 6.699,98$ & R\$ 74.513,81 \\
\hline
\end{tabular}


Elaborou-se o demonstrativo de resultado financeiro a partir do novo processo de impressão de exames proposto com a utilização de papel fotográfico.

A Tabela 8 ilustra o demonstrativo de resultado financeiro.

Tabela 8 - Resultado financeiro - novo processo (papel fotográfico)

\begin{tabular}{lc}
\hline Descrição & Valor \\
\hline Receita & $\mathrm{R} \$ 640.300,02$ \\
Custo Fixo & $\mathrm{R} \$ 111.558,58$ \\
Custo Variável (médico) & $\mathrm{R} \$ 255.219,55$ \\
Custo variável (insumos) & $\mathrm{R} \$ 74.513,81$ \\
\hline Resultado & $\mathbf{R} \$ \mathbf{1 9 9 . 0 0 8 , 0 9}$ \\
\hline Resultado Mensal & $\mathbf{R} \$ \mathbf{1 6 . 5 8 4 , 0 1}$ \\
\hline \multicolumn{2}{c}{ Fonte: Elaborado pelo autor, 2018. }
\end{tabular}

\section{EVIDÊNCIAS DA ANÁLISE FINANCEIRA}

A partir da análise realizada no período de janeiro a dezembro do ano de 2017 (dois mil e dezessete), levando em consideração as seguintes variáveis: quantidade de exames realizados, receita bruta, custos fixos, custos variáveis e proposta de locação de impressora para impressão de exames em papel fotográfico, chegou-se ao resultado financeiro (comparativo) descrito na Tabela 9.

Tabela 9 - Resultado financeiro - filme radiológico $x$ papel fotográfico

\begin{tabular}{lcc}
\hline Descrição & Valor & Resultado \\
\hline & $\mathrm{R} \$$ & \\
Resultado - Processo Atual & $168.125,11$ & $18,37 \%$ \\
& $\mathrm{R} \$$ & \\
Resultado - Novo Processo & $199.008,09$ & \\
\hline
\end{tabular}

Fonte: Elaborado pelo autor, 2018.

\section{CONSIDERAÇÕES FINAIS}

O presente estudo de viabilidade financeira surgiu pela necessidade de melhoria dos resultados do serviço de diagnóstico por imagem e para auxiliar os gestores no processo de tomada de decisão. Com isso a pesquisa se propôs a avaliar o impacto financeiro da mudança do processo de impressão de exames em serviço de diagnóstico por imagem em hospital de pequeno porte do Estado do Rio Grande do Sul.

Conclui-se com o estudo, que a mudança no processo de impressão de exames substituindo o filme radiológico pelo papel fotográfico, é viável, onde no período pesquisado, representaria um ganho superior a $\mathrm{R} \$ 30.000,00$ (trinta mil reais), o que representa um incremento no resultado financeiro do serviço de 18,37\%. Recomenda-se a instituição hospitalar realizar a locação da impressora para impressão dos exames em papel fotográfico.

Sugere-se para estudos futuros, avaliar o impacto financeiro considerando as perspectivas de mercado e o desperdício na impressão de exames.

\section{REFERÊNCIAS}

ABDULLAH, Lazim. Fuzzy multi criteria decision making and its applications: a brief review of category. Procedia-Social and Behavioral Sciences, v. 97, p. 131-136, 2013.

ASSAF NETO, Alexandre; LIMA, Fabiano Guasti. Fundamentos de Administração Financeira. 2 ed. São Paulo: Editora Atlas, 2014.

BISAGNI, C. Unidade de Imagem: a inserção da consulta de enfermagem na qualificação do processo de trabalho. 2000. Dissertação (Mestrado em Enfermagem), Faculdade de Enfermagem da Universidade Estadual do Rio de Janeiro, Rio de Janeiro.

BORBA, Gustavo Severo de; NETO, Francisco José Kliemann. Gestão hospitalar: identificação das práticas de aprendizagem existentes em hospitais. Revista Saúde e Sociedade. v. 17, n. 1, p. 44-60, 2008. 
CABRELLI, Fantini Lígia; FERREIRA, Ademilson. 2007. Contabilidade gerencial como ferramenta no processo de tomada de decisão. Revista cientifica eletrônica de ciências contábeis. Ano V, n.9.

DACORSO, A. L. R. Tomada de decisão e Risco: a administração da inovação em pequenas indústrias químicas. 2000. 236 f. Dissertação (Mestrado em Administração) Faculdade de Economia, Administração e Contabilidade, Universidade de São Paulo USP, São Paulo.

GITMAN, Lawrence J. Princípios de administração financeira. São Paulo: Pearson, 2004.

Herrera-Viedma, E. García-Lapresta, J.L. Information fusion in consensus and decision making. Information Fusion, 17, 2-3, 2014.

JUNG, Carlos Fernando. Metodologia para pesquisa e desenvolvimento - aplicada a novas tecnologias, produtos e processos. Rio de Janeiro: Axcel books do Brasil Editora Ltda, 2004.

RAMOS, S. C.; TAKAHASHI, A. R. W.; ROGLIO, K. D. Análise da Produção Nacional sobre Processo Decisório no Período de 2004-2014. Contextus - Revista Contemporânea de Economia e Gestão, v. 13, n. 3, p. 156-184, 2015.

SILVA Jorge Luis Samartin de Souza e. A tentativa da profissionalização do gestor público hospitalar: o caso da secretaria de saúde do Estado do Piauí. 2014. 106 f. Dissertação (Mestrado Profissional em Saúde Pública) - Programa de Pós-Graduação em Saúde Pública, Centro de Pesquisas Aggeu Magalhães, Fundação Oswaldo Cruz, Recife.

ZAMARIOLI, R. Participação e tomada de decisão na pequena empresa: estudo multi-casos em três empresas industriais do ramo metalúrgico de Ribeirão Preto - SP. Dissertação de Mestrado, Escola de Engenharia de São Carlos, USP, São Carlos, 2003.

\section{Jocias Maier Zanatta}

Doutorando em Administração pela Universidade Federal de Santa Maria - UFSM, Mestre em Desenvolvimento pela Universidade Regional do Noroeste do Estado do Rio Grande do Sul - UNIJUI, Especialização em Gestão Financeira, Controladoria e Auditoria e Graduação em Administração pela Sociedade Educacional Três de Maio - SETREM. Professor e Administrador hospitalar. 